\title{
Homocisteína y otros factores de riesgo cardiovascular en niños de educación básica primaria del Colegio Distrital Manuel Elkin Patarroyo, Bogotá, D.C.- Colombia. Estudio piloto
}

\author{
Homocysteine and other Cardiovascular Risk Factors in Elementary \\ School Children of the School District "Manuel Elkin Patarroyo", \\ Bogota, D.C. - Colombia. Pilot study
}

Título abreviado: Homocisteína y otros factores de riesgo cardiovascular en niños

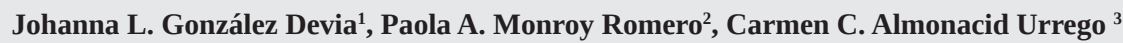

\section{Resumen}

Objetivo. Evaluar los niveles de homocisteína total (tHcy) y su asociación con otros factores de riesgo cardiovascular (FRCV) en niños de educación básica primaria del Colegio Manuel Elkin Patarroyo. Método. Estudio descriptivo en 50 niños de 6 a 12 años, seleccionados mediante muestreo no probabilístico por conveniencia. Se cuantificaron niveles de tHcy, lípidos y glucosa, se midió presión arterial y se tomaron medidas antropométricas. Los estilos de vida se determinaron con formatos validados y se estableció el punto de corte para diagnosticar hiperhomocisteinemia (HHcy) en la población. Resultados. Las niñas presentaron concentraciones mayores de triglicéridos y c-VLDL que los niños $(p=0.05)$. El valor medio de tHcy para la población general fue de $5.0( \pm 1,15) \mu \mathrm{mol} / \mathrm{L}$ y el punto de corte para HHcy $6.92 \mu \mathrm{mol} / \mathrm{L}$. Presentaron HHcy $7.7 \%$ de las niñas y $12,5 \%$ de los niños. Del grupo con HHcy, 20\% estaba en sobrepeso, 40\% presentaba hipertensión, $20 \%$ expresó c-HDL disminuido, $62 \%$ tenía un consumo bajo de carne y $80 \%$ alta ingesta de hamburguesas, papas fritas y refrescos. Conclusiones. Aunque la HHcy no estuvo asociada con otros FRCV, es necesario implementar programas que permitan modificar estilos de vida inadecuados.

Palabras claves: Homocisteína, hiperhomocisteinemia, factor de riesgo cardiovascular, enfermedad cardiovascular, niñez. 


\begin{abstract}
Objective. To assess levels of total homocysteine (tHcy) and its association with other cardiovascular risk factors (CRF) in elementary school children Manuel Elkin Patarroyo College. Methods. Descriptive study in 50 children aged 6 to 12 years, selected through non-probability convenience sampling. Levels of tHcy, lipids and glucose were measured, blood pressure was measured and anthropometric measurements were taken. The lifestyles were determined with validated formats and the cutoff was established to diagnose hyperhomocysteinemia (HHcy) in the population. Results. Girls had higher concentrations of triglycerides and VLDL-c than boys $(p=0.05)$. The mean tHcy in the general population was $5.0( \pm 1.15) \mu \mathrm{mol} / \mathrm{L}$ and the cutoff for HHcy was $6.92 \mu \mathrm{mol} / \mathrm{L} .7 .7 \%$ of girls and $12.5 \%$ of the boys had HHcy. Among this group, $20 \%$ were overweight, 40\% had hypertension, 20\% expressed decrease c-HDL, 62\% (31) had a low intake of meat, and $80 \%$ (4) high intake of hamburger, fries and soda. Conclusion. Although HHcy was not associated with other CVRF, it is necessary to implement programs that allowto modify inappropriate lifestyles.
\end{abstract}

Keywords: Homocysteine, hyperhomocysteinemia, factor of cardiovascular risk, cardiovascular disease, childhood.

\title{
Introducción
}

En los últimos años, la enfermedad cardiovascular (ECV) se ha convertido en una de las principales causas de muerte a nivel mundial (1). En Colombia, las muertes por ECV se han incrementado en la última década. En el año 2000 la tasa por enfermedad isquémica del corazón fue de 55,61 por cada cien mil habitantes y en el 2008 ascendió a 64,45 muertes por cada cien mil habitantes (1).

En la población infantil se ha identificado la presencia de factores de riesgo para ECV tales como cambios en los hábitos alimentarios y disminución de actividad física, especialmente en población urbana (2), y el incremento de otros factores como tabaquismo, alcoholismo y consumo de drogas; factores todos que conllevan al aumento de este tipo de patologías.

Dentro de los factores de riesgo emergentes se incluye como factor de riesgo independiente, la hiperhomocisteinemia (HHcy) (3). Existen numerosas evidencias que señalan el papel aterogénico de ésta y que muestran que el incremento en la concentración de la homocisteína sérica está asociado con un mayor riesgo de enfermedades cerebrovasculares, cardiovasculares o neurológicas (4). Así mismo, se conoce que el proceso ateroesclerótico se inicia en la infancia y que el grado de extensión de las lesiones en niños y adultos jóvenes se correlaciona con la presencia de los mismos factores de riesgo identificados en adultos (5).

Si la hiperhomocisteinemia se detecta desde la infancia, la determinación de la prevalencia como intervención primaria de ésta en nińos y nińas cobra especial importancia para implementar programas de promoción y prevención enfocados a ajustar y mejorar sus estilos de vida (6), contribuyendo así con la creación de factores protectores que disminuyan los efectos negativos sobre la salud y el bienestar, minimizando a la vez su perfil de riesgo $(7,8)$ y disminuyendo las futuras tasas de mortalidad por ECV. Como consecuencia, se obtiene mejora en la calidad de vida de los niños y disminución de los costos adicionales que para el sistema de salud genera el incremento de la patología cardiovascular. Sin embargo, existen pocos estudios a nivel mundial y nacional que aborden 
la prevalencia de la hiperhomocisteinemia en población infantil.

Bajo este contexto, el objetivo del presente estudio fue evaluar los niveles basales séricos de homocisteína total y su asociación con otros factores de riesgo cardiovascular en niños de educación básica primaria del colegio Manuel Elkin Patarroyo, Localidad Santa Fe, de Bogotá D.C. (Colombia).

\section{Materiales y métodos}

La presente investigación corresponde a un estudio de corte transversal en el que se incluyeron 50 niños con edades comprendidas entre los 6 y los 12 años que cursaban educación básica primaria en el colegio Manuel Elkin Patarroyo de Bogotá D.. (Colombia). La participación fue voluntaria y se autorizó con la firma del consentimiento informado por parte de los padres o acudientes.

A cada uno de los participantes se le aplicó una encuesta de recolección de datos basada en formatos validados (historia dietaria para alimentación, AUDIT para consumo de alcohol y Test de Fagerström para tabaquismo) (9-10). Se tomó una muestra de sangre total con ayuno previo de 12 horas para determinar los niveles séricos de lípidos (c-total, c-HDL, c-LDL, c-VLDL, triglicéridos) y glucosa, y otra muestra de sangre heparinizada para la cuantificación de tHcy. Así mismo, se obtuvieron medidas antropométricas (IMC y porcentaje de grasa corporal) por bioimpedancia eléctrica, y se tomó la presión arterial por esfingomanometría. En aquellos niños en los que se obtuvo un dato de tensión arterial por encima de los parámetros normales para el momento de la toma de muestra, se realizaron tres lecturas posteriores en un día diferente con intervalos de 5 minutos y se dejó como valor definitivo el valor más bajo de los tres (11, 12).

Para la clasificación de los parámetros a analizar se utilizaron los siguientes consensos internacionales: World Health Organization para rango de edad, medidas antropométricas y curvas de crecimiento para sobrepeso y obesidad (13); criterios establecidos por el Programa Nacional e Internacional de Educación sobre Colesterol para Niños (ATP III), para lípidos sanguíneos (14); guías de diagnóstico y tratamiento de hipertensión en niños de la $\mathrm{Na}$ tional Heart, Lung and Blood Institute (NHLBI) y el National High Blood Pressure Education Program (NHBPEP), para tensión arterial (15) y criterios establecidos por la Asociación Latinoamericana de Diabetes (ALAD) para glicemia (16). El punto de corte para HHcy se estableció con base en el valor obtenido para el percentil 90 de la población. La cuantificación de los parámetros bioquímicos se realizó en el autoanalizador MINDRAY - BS200, propiedad de ANNAR Diagnóstica, mediante pruebas colorimétricas Spin React.

Todas las decisiones y el desarrollo de este trabajo de investigación se basaron en los principios éticos y juicios de valor moral de las investigaciones médicas en seres humanos presentados en la declaración de Helsinki, versión $64^{\mathrm{a}}$, Fortaleza-Brasil, 2013. Igualmente, se tuvo en cuenta el código bioético de Bacteriólogos y los aspectos éticos especificados en la resolución Nro. 008430 de 1993, título II, capítulo I ("de los aspectos éticos de la investigación en seres humanos”) del Ministerio de la Protección Social de Colombia.

\section{Análisis estadístico}

Inicialmente se evaluó la distribución de normalidad de las variables. Las variables con distribución normal se describen con el promedio y la desviación estándar (SD) y las que no, con la mediana y el rango. Para las variables categóricas se presentan los porcentajes.

Las variables del perfil lipídico, medidas antropométricas, tensión arterial y homocisteína se analizaron como variables continuas y categóricas y las variables relacionadas con la dieta como variables ordinales. 
La homocisteína se analizó como variable categórica $(\leq 10,0 \mu \mathrm{mol} / \mathrm{L})$ y como variable continua; además, se estableció el percentil 90 como punto de corte de la distribución de la homocisteína de los niños incluidos en el estudio.

Para establecer la diferencia en la distribución de dos variables numéricas se utilizó la prueba "t de Student" para las variables con distribución normal y la prueba de Mann-Whitney para las variables con distribución no normal. En las variables continuas con distribución normal con más de dos categorías se utilizó la prueba ANOVA. La diferencia en la distribución de las variables categóricas se estableció con la prueba Ji al cuadrado de diferencia y con la prueba de Fisher cuando el análisis fue de tablas de $2 \mathrm{X} 2$ con un valor esperado menor a cinco.

De acuerdo con el percentil 90 y para las variables de sexo, perfil lipídico, medidas antropométricas, tensión arterial y dieta, la homocisteína se analizó como variable categórica, menor o igual a 6,92 $\mu \mathrm{mol} / \mathrm{L}$ y mayor a $6.92 \mu \mathrm{mol} / \mathrm{L}$. Se consideró como significativo un valor con $p \leq 0,05$.

\section{Resultados}

\section{Características Generales}

El promedio de edad en la población general fue $9,3 \pm 1,6$ años. Hubo predominio del género femenino $(52 \%)$ con una relación mujer/hombre de $1: 1$.

\section{Factores de riesgo cardiovascular Datos bioquímicos}

Como se observa en la Tabla 1, la totalidad de la población tuvo niveles basales de glucosa sérica dentro del rango considerado normal. Se presentó $52 \%$ de cualquier tipo de dislipidemias, distribuidas estas de la siguiente manera: hipercolesterolemia 27\%, hipertrigliceridemia $8 \%$, disminución en los niveles de c-HDL 27\%, incremento en las concentraciones de c-LDL $30 \%$ e incremento en las concentraciones de c-VLDL 8\%. La distribución para los valores de triglicéridos y c-VLDL demostró diferencia por género, observándose concentraciones mayores en las niñas que en los niños $(p=0.05)$.

Tabla 1. Descripción de las variables bioquímicas y antropométricas.

\begin{tabular}{|c|c|c|c|c|c|c|}
\hline \multirow{2}{*}{\multicolumn{2}{|c|}{ Variable }} & \multicolumn{2}{|l|}{ Niños } & \multicolumn{2}{|l|}{ Niñas } & \multirow[b]{2}{*}{$\mathrm{p}$} \\
\hline & & $\mathrm{n}$ & $\%$ & $\mathrm{n}$ & $\%$ & \\
\hline \multirow{2}{*}{ Glucosa } & Normal & 24 & 100.0 & 26 & 100.0 & \multirow{2}{*}{ NA } \\
\hline & Alta & 0 & 0.0 & 0 & 0.0 & \\
\hline \multirow{2}{*}{ Triglicéridos } & Normal & 23 & 95.8 & 25 & 96.2 & \multirow{2}{*}{$1.00^{*}$} \\
\hline & Altos & 1 & 4.2 & 1 & 3.8 & \\
\hline \multirow{3}{*}{$\mathrm{t}$-Colesterol } & Aceptable & 12 & 50.0 & 13 & 50.0 & \multirow{3}{*}{ NA } \\
\hline & Limítrofe & 8 & 33.3 & 10 & 38.5 & \\
\hline & Alto & 4 & 16.7 & 3 & 11.5 & \\
\hline
\end{tabular}




\begin{tabular}{|c|c|c|c|c|c|c|}
\hline & Normal & 21 & 87.5 & 22 & 84.6 & \\
\hline & Bajo & 3 & 12.5 & 4 & 15.4 & \\
\hline \multirow{3}{*}{ c-LDL } & Aceptable & 10 & 41.7 & 143 & 50.0 & \multirow{3}{*}{ NA } \\
\hline & Limítrofe & 10 & 41.7 & 9 & 34.6 & \\
\hline & Alto & 4 & 16.7 & 4 & 15.4 & \\
\hline \multirow{2}{*}{ c-VLDL } & Normal & 23 & 95.8 & 25 & 96.2 & \multirow{2}{*}{$1.00^{*}$} \\
\hline & Alto & 1 & 4.2 & 1 & 3.8 & \\
\hline \multirow{2}{*}{$\begin{array}{l}\text { Homocisteína } \\
\text { Percentil } 90\end{array}$} & $\leq 6.92 \mu \mathrm{mol} / \mathrm{L}$ & 21 & 87.5 & 24 & 92.3 & \multirow{2}{*}{$0.66^{*}$} \\
\hline & $>6.92 \mu \mathrm{mol} / \mathrm{L}$ & 3 & 12.5 & 2 & 7.7 & \\
\hline \multirow{3}{*}{ Presión arterial } & Normal & 33 & 73,3 & 3 & 60 & \multirow{3}{*}{ NA } \\
\hline & Alta & 9 & 20 & 2 & 40 & \\
\hline & Baja & 3 & 6,7 & 0 & 0 & \\
\hline \multirow{4}{*}{ IMC } & Normal & 37 & 82,2 & 4 & 80 & \multirow{4}{*}{ NA } \\
\hline & Sobrepeso & 4 & 8,9 & 1 & 20 & \\
\hline & Obesidad & 2 & 4,4 & 0 & 0 & \\
\hline & Bajo peso & 2 & 4,4 & 0 & 0 & \\
\hline \multirow{4}{*}{$\begin{array}{l}\text { Porcentaje de } \\
\text { grasa corporal }\end{array}$} & Óptimo & 27 & 60 & 2 & 40 & \multirow{4}{*}{ NA } \\
\hline & Moderadamente alto & 10 & 22,2 & 2 & 40 & \\
\hline & Alto & 3 & 6,7 & 0 & 0 & \\
\hline & Muy alto & 5 & 11,1 & 1 & 20 & \\
\hline
\end{tabular}

p: Diferencias de proporciones

*Test de Fisher

En relación con la Hcy, el valor medio de tHcy para la población general fue de 5,0 $1,15 \mu \mathrm{mol} / \mathrm{L}$, sin encontrarse diferencias por género en su distribución, Figura 1. El percentil 90 para homocisteína en la población fue $\leq 6.92 \mu \mathrm{mol} / \mathrm{L}$, valor que se estableció como el punto de corte para diagnosticar HHcy en los niños. Con base en él se demostró que 10\% de los escolares presentaban HHcy. De ellos, $7.7 \%$ fueron niñas y $12.5 \%$ niños, sin encontrarse diferencia en la distribución porcentual entre los dos sexos, Tabla 2. 


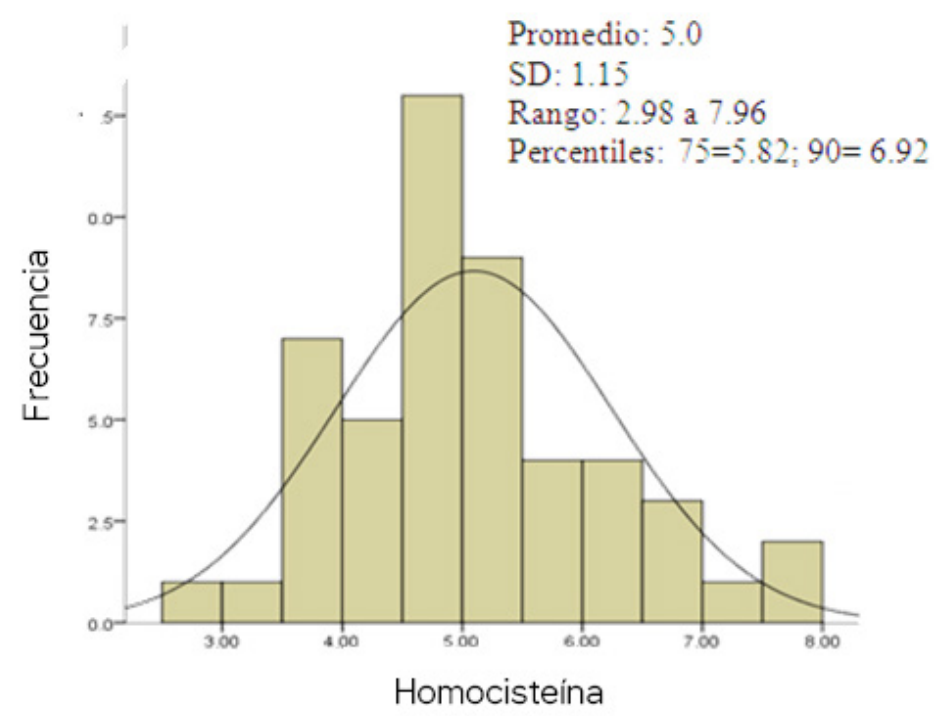

Figura 1. Distribución de homocisteína en el grupo de niños estudiados.

Tabla 2. Distribución de la homocisteína, percentil 90, según variables bioquímicas y antropométricas.

\begin{tabular}{|c|c|c|c|c|c|c|}
\hline \multirow{3}{*}{ Variable } & & \multicolumn{5}{|c|}{ Homocisteína (Hcy) } \\
\hline & & \multicolumn{2}{|l|}{$\leq 6,92 \mu \mathrm{mol} / \mathrm{L}$} & \multicolumn{2}{|l|}{$>6,92 \mu \mathrm{mol} / \mathrm{L}$} & \multirow[b]{2}{*}{$\mathrm{p}$} \\
\hline & & No. & $\%$ & No. & $\%$ & \\
\hline \multirow{2}{*}{ Glucosa } & Normal & 45 & 90,0 & 5 & 10,0 & \multirow{2}{*}{ NA } \\
\hline & Alta & 0 & 0 & 0 & 0 & \\
\hline \multirow{2}{*}{ Triglicéridos } & Normal $<200 \mathrm{mg} / \mathrm{dL}$ & 43 & 95.6 & 5 & 100,0 & \multirow{2}{*}{$1.00^{*}$} \\
\hline & Altos & 2 & 4,4 & 0 & - & \\
\hline \multirow{3}{*}{ Colesterol total } & $\begin{array}{l}\text { Aceptable } \leq 170 \\
\mathrm{mg} / \mathrm{dL}\end{array}$ & 20 & 44,4 & 5 & 100,0 & \multirow{3}{*}{ NA } \\
\hline & Limítrofe & 18 & 40 & 0 & 0,0 & \\
\hline & Alto & 7 & 15,6 & 0 & 0,0 & \\
\hline \multirow{2}{*}{ HDL } & Normal $\geq 40 \mathrm{mg} / \mathrm{dL}$ & 39 & 86,7 & 4 & 80,0 & \multirow{2}{*}{$1.00^{*}$} \\
\hline & Bajo & 6 & 13,3 & 1 & 20,0 & \\
\hline \multirow{3}{*}{ LDL } & $\begin{array}{l}\text { Aceptable } \leq 100 \\
\mathrm{mg} / \mathrm{dL}\end{array}$ & 18 & 40 & 5 & 100 & \multirow{3}{*}{ NA } \\
\hline & Limítrofe & 19 & 42,2 & 0 & 0,0 & \\
\hline & Alto & 8 & 17,8 & 0 & 0,0 & \\
\hline
\end{tabular}




\begin{tabular}{|c|c|c|c|c|c|c|}
\hline \multirow{2}{*}{ VLDL } & Normal $<40 \mathrm{mg} / \mathrm{dL}$ & 43 & 95,6 & 5 & 100,0 & \multirow{2}{*}{$1.00^{*}$} \\
\hline & Alto & 2 & 4,4 & 0 & 0,0 & \\
\hline \multirow{4}{*}{ IMC } & Normal & 37 & 82,2 & 4 & 80,0 & \multirow{4}{*}{$\mathrm{N} A$} \\
\hline & Sobrepeso & 4 & 8,9 & 1 & 20,0 & \\
\hline & Obesidad & 2 & 4,4 & 0 & 0,0 & \\
\hline & Bajo peso & 2 & 4,4 & 0 & 0,0 & \\
\hline \multirow{4}{*}{$\begin{array}{l}\text { Porcentaje } \\
\text { grasa corporal }\end{array}$} & Óptimo & 27 & 60,0 & 2 & 40,0 & \multirow{4}{*}{ NA } \\
\hline & Moderadamente alto & 10 & 22,2 & 2 & 40,0 & \\
\hline & Alto & 3 & 6,7 & 0 & 0,0 & \\
\hline & Muy alto & 5 & 11,1 & 1 & 20,0 & \\
\hline \multirow{3}{*}{$\begin{array}{l}\text { Presión } \\
\text { arterial para el } \\
\text { momento de la } \\
\text { toma }\end{array}$} & Normal & 33 & 73,3 & 3 & 60,0 & \multirow{3}{*}{ NA } \\
\hline & Alta & 9 & 20,0 & 2 & 40,0 & \\
\hline & Baja & 3 & 6,7 & 0 & 0,0 & \\
\hline
\end{tabular}

p: Diferencias de proporciones

*Test de Fisher

Aunque no se demostró asociación directa entre los niveles de tHcy y las otras variables estudiadas (Tabla 2 y 3), es importante resaltar que 80\% de los niños con HHcy consumía refrescos y golosinas más de una vez al día y hamburguesas y papas fritas algunas veces a la semana, Tabla 3. Asímismo, $20 \%$ estaba en sobrepeso y tenía bajas concentraciones de c-HDL y $40 \%$ presentó presión arterial alta al momento de la toma de muestra, Tabla 1 .

Tabla 3. Distribución de la homocisteína percentil 90 según ingesta dietética.

\begin{tabular}{|c|c|c|c|c|c|}
\hline \multirow{3}{*}{ Variable } & & \multicolumn{4}{|c|}{ Homocisteína (Hcy) } \\
\hline & & \multicolumn{2}{|c|}{$\leq 6,92 \mu \mathrm{mol} / \mathrm{L}$} & \multicolumn{2}{|c|}{$>6,92 \mu \mathrm{mol} / \mathrm{L}$} \\
\hline & & No. & $\%$ & No. & $\%$ \\
\hline \multirow{5}{*}{ Café } & Mas una vez día & 4 & 8,9 & 1 & 20 \\
\hline & Una vez al día & 12 & 26,7 & 0 & 0 \\
\hline & Algunas veces a la semana & 18 & 40 & 2 & 40 \\
\hline & Algunas veces al mes & 8 & 17,8 & 2 & 40 \\
\hline & Nunca & 3 & 6,7 & 0 & 0 \\
\hline
\end{tabular}




\begin{tabular}{|c|c|c|c|c|c|}
\hline \multirow{5}{*}{ Leche-batidos } & Mas una vez día & 8 & 17,8 & 1 & 20 \\
\hline & Una vez al día & 12 & 26,7 & 2 & 40 \\
\hline & Algunas veces a la semana & 15 & 33,3 & 0 & 0 \\
\hline & Algunas veces al mes & 8 & 17,8 & 0 & 0 \\
\hline & Nunca & 2 & 4,4 & 2 & 40 \\
\hline \multirow{5}{*}{ Jugos } & Mas una vez día & 17 & 37,8 & 2 & 40 \\
\hline & Una vez al día & 13 & 28,9 & 1 & 20 \\
\hline & Algunas veces a la semana & 11 & 24,4 & 0 & 0 \\
\hline & Algunas veces al mes & 2 & 4,4 & 2 & 40 \\
\hline & Nunca & 2 & 4,4 & 0 & 0 \\
\hline \multirow{5}{*}{ Hamburguesas y salchichas } & Mas una vez día & 2 & 4,4 & 0 & 0 \\
\hline & Una vez al día & 1 & 2,2 & 0 & 0 \\
\hline & Algunas veces a la semana & 17 & 37,8 & 4 & 80 \\
\hline & Algunas veces al mes & 14 & 31,1 & 1 & 20 \\
\hline & Nunca & 11 & 24,4 & 0 & 0 \\
\hline \multirow{5}{*}{ Papas fritas o de paquete } & Mas una vez día & 1 & 2,2 & 2 & 40 \\
\hline & Una vez al día & 10 & 22,2 & 0 & 0 \\
\hline & Algunas veces a la semana & 21 & 46,7 & 3 & 60 \\
\hline & Algunas veces al mes & 11 & 24,4 & 0 & 0 \\
\hline & Nunca & 2 & 4,4 & 0 & 0 \\
\hline \multirow{5}{*}{ Pasteles y dulces } & Mas una vez día & 9 & 20 & 3 & 60 \\
\hline & Una vez al día & 13 & 28,9 & 0 & 0 \\
\hline & Algunas veces a la semana & 10 & 22,2 & 0 & 0 \\
\hline & Algunas veces al mes & 10 & 22,2 & 2 & 40 \\
\hline & Nunca & 3 & 6,7 & 0 & 0 \\
\hline
\end{tabular}




\begin{tabular}{|c|c|c|c|c|c|}
\hline \multirow{5}{*}{ Verduras y hortalizas } & Mas una vez día & 5 & 11,1 & 0 & 0 \\
\hline & Una vez al día & 15 & 33,3 & 0 & 0 \\
\hline & Algunas veces a la semana & 16 & 35,6 & 4 & 80 \\
\hline & Algunas veces al mes & 3 & 6,7 & 0 & 0 \\
\hline & Nunca & 6 & 13,3 & 1 & 20 \\
\hline \multirow{5}{*}{ Frutas } & Mas una vez día & 11 & 24,4 & 2 & 40 \\
\hline & Una vez al día & 14 & 31,1 & 1 & 20 \\
\hline & Algunas veces a la semana & 17 & 37,8 & 2 & 40 \\
\hline & Algunas veces al mes & 2 & 4,4 & 0 & 0 \\
\hline & Nunca & 1 & 2,2 & 0 & 0 \\
\hline \multirow{5}{*}{ Golosinas } & Mas una vez día & 11 & 24,4 & 4 & 80 \\
\hline & Una vez al día & 7 & 15,6 & 0 & 0 \\
\hline & Algunas veces a la semana & 10 & 22,2 & 1 & 20 \\
\hline & Algunas veces al mes & 13 & 28,9 & 0 & 0 \\
\hline & Nunca & 4 & 8,9 & 0 & 0 \\
\hline \multirow{5}{*}{ Legumbres } & Mas una vez día & 7 & 15,6 & 0 & 0 \\
\hline & Una vez al día & 5 & 11,1 & 1 & 20 \\
\hline & Algunas veces a la semana & 25 & 55,6 & 4 & 80 \\
\hline & Algunas veces al mes & 5 & 11,1 & 0 & 0 \\
\hline & Nunca & 3 & 6,7 & 0 & 0 \\
\hline \multirow{5}{*}{ Yogurt } & Mas una vez día & 9 & 20 & 1 & 20 \\
\hline & Una vez al día & 11 & 24,4 & 2 & 40 \\
\hline & Algunas veces a la semana & 20 & 44,4 & 1 & 20 \\
\hline & Algunas veces al mes & 4 & 8,9 & 1 & 20 \\
\hline & Nunca & 1 & 2,2 & 0 & 0 \\
\hline
\end{tabular}




\begin{tabular}{|c|c|c|c|c|c|}
\hline \multirow{5}{*}{ Queso } & Mas una vez día & 7 & 15,6 & 0 & 0 \\
\hline & Una vez al día & 8 & 17,8 & 0 & 0 \\
\hline & Algunas veces a la semana & 16 & 35,6 & 4 & 80 \\
\hline & Algunas veces al mes & 13 & 28,9 & 1 & 20 \\
\hline & Nunca & 1 & 2,2 & 0 & 0 \\
\hline \multirow{5}{*}{ Carne } & Todos los días & 8 & 17,8 & 0 & 0 \\
\hline & 5 a 6 veces por semana & 5 & 11,1 & 0 & 0 \\
\hline & 3 a 4 veces por semana & 11 & 24,4 & 2 & 40 \\
\hline & 1 a 2 veces por semana & 16 & 35,6 & 3 & 60 \\
\hline & Rara vez o nunca & 5 & 11,1 & 0 & 0 \\
\hline \multirow{4}{*}{ Pescado } & Todos los días & 1 & 2,2 & 0 & 0 \\
\hline & 5 a 6 veces por semana & 2 & 4,4 & 1 & 20 \\
\hline & 1 a 2 veces por semana & 12 & 26,7 & 0 & 0 \\
\hline & Rara vez o nunca & 30 & 66,7 & 4 & 80 \\
\hline \multirow{5}{*}{ Huevo } & Todos los días & 19 & 42,2 & 1 & 20 \\
\hline & 5 a 6 veces por semana & 2 & 4,4 & 0 & 0 \\
\hline & 3 a 4 veces por semana & 11 & 24,4 & 2 & 40 \\
\hline & 1 a 2 veces por semana & 10 & 22,2 & 2 & 40 \\
\hline & Rara vez o nunca & 3 & 6,7 & 0 & 0 \\
\hline
\end{tabular}

\section{Alcohol y tabaco}

El análisis de la variable tabaquismo mostró que aunque la mayoría de la población $(97.8 \%)$ no ha probado el cigarrillo, un niño $(2.2 \%)$ ya lo había hecho a la edad de 8 ańos. En relación con la ingesta de licor, la mayoría de los niños $(80 \%)$ manifestó nunca haber ingerido algún tipo de bebida alcohólica. De los 9 que admitieron haberla probado, tres lo hicieron a la edad de 6 ańos y el resto entre los 8 y 9 años.

\section{Hábitos alimentarios}

La ingesta dietética en relación con la homocisteína se describe en la Tabla 3. Los alimentos de consumo más frecuente (ingeridos al menos una vez por día), fueron: huevos (40\%); verduras, 
hortalizas y frutas (30\%); leche y jugos (28\%); café $(24 \%)$; pasteles, dulces y yogurt $(26 \%)$ y alimentos como embutidos, papas fritas y paquetes (20\%). La carne se incluyó en la dieta de 1 o 2 veces por semana $(38 \%)$ y la mayoría de la población rara vez o nunca consume pescado (68\%).

\section{Datos antropométricos}

La clasificación de la población con base en el IMC demostró que $16.7 \%$ de los niños y $3.8 \%$ de las niñas estaban en sobrepeso y tan solo dos niños $(8.3 \%)$ eran obesos. De manera concordante, mientras la totalidad de las niñas exhibió porcentajes de grasa óptimos, 8,3\% de los niños tuvo porcentajes de grasa altos y $25 \%$ muy altos (Tabla 1).

\section{Tensión arterial}

La medición de la tensión arterial demostró que $22 \%$ de los escolares tenía presión arterial alta y $6 \%$ baja al momento de la toma de muestra (Tabla 1$)$.

\section{Discusión}

A pesar de que datos estadísticos de la ONU, la OEA, la Organización Mundial de la Salud y varias organizaciones privadas colombianas confirman que Colombia es el país latinoamericano donde se inicia el consumo de bebidas alcohólicas y tabaco en edades más tempranas y señalan que la edad promedio en que los colombianos comienzan a beber y a fumar cigarrillos es a los 12 y 14 ańos respectivamente (17-20), nuestro estudio encontró edades mucho menores; 6 años para alcohol y 8 ańos para cigarrillo, esto puede deberse a que el colegio es de carácter público con un menor nivel socioeconómico.

Estos hallazgos ponen de manifiesto la necesidad de realizar verificaciones posteriores que comprueban si es necesario implementar programas de prevención que aborden edades más tempranas de las actualmente referidas, que permitan a la vez generar factores protectores en la población y pre- venir los riesgos ya demostrados del consumo de licor y tabaco durante la infancia y la adolescencia tales como alteración del desarrollo del sistema nervioso central y afectación del crecimiento y la función pulmonar (20-22).

Con respecto a las concentraciones de tHcy en niños, son pocos los estudios que han investigado sobre ello. Debido a esto, el presente trabajo pretende establecer los valores propios de tHcy de una población escolar colombiana, con el fin de explorar la relación entre tHcy y ECV en este grupo etario.

El valor medio de tHcy de nuestra población $(5,0 \pm 1,15 \mu \mathrm{mol} / \mathrm{L})$, es un poco más bajo que el observado en escolares de cinco departamentos de la región oriental de Colombia $(6,3 \pm 0,25 \mu \mathrm{mol} / \mathrm{L})$ (23) y varía con respecto a los encontrados en otras poblaciones. A nivel de Latinoamérica los estudios muestran concentraciones de $9.78 \pm 1.73 \mu \mathrm{mol} / \mathrm{L}$ para niños mexicanos (24) y de $7.3 \pm 1,3 \mu \mathrm{mol} / \mathrm{L}$ para escolares brasileños, en quienes se observaron concentraciones mayores en los varones que en las mujeres con incremento con respecto a la edad, y promedios más altos en los estudiantes de colegios públicos (20).

En Norteamérica se han encontrado concentraciones de $4.01 \mu \mathrm{mol} / \mathrm{L}(25)$ en nińos canadienses y de $4,36 \mu \mathrm{mol} / \mathrm{L}$ en niños estadounidenses con edades comprendidas entre los 8 y los 11 ańos (26) observándose incrementos proporcionales con respecto a la edad. En relación a Europa, se han descrito concentraciones plasmáticas de tHcy de 6.2 (5.6 - 6.9) $\mu \mathrm{mol} / \mathrm{L}$ para población holandesa (27) y de $7.6 \mu \mathrm{mol} / \mathrm{L}$ para escolares checos (28). Estas divergencias pueden deberse a diferencias en los estilos de vida y en los hábitos alimenticios propios de cada región; en especial, las variaciones en la ingesta diaria de folatos, vitamina B12 y vitamina B6, variable que no fue incluida en el presente estudio.

De igual manera, es importante considerar las diferencias entre las metodologías utilizadas para la 
cuantificación de tHcy en cada uno de los estudios: EIA, HPLC, QL y colorimetría, que permiten hacer hincapié en la necesidad de la estandarización de estas pruebas.

Con respecto a la dieta, el consumo de frutas, verduras y hortalizas es relativamente bajo en la población estudiada (una vez a la semana), datos que son similares a los resultados mostrados en la encuesta nacional de nutrición -ENSIN- (2010), donde se observó que más de la mitad de los nińos entre 5 y 13 años consumen fruta, verduras y hortalizas solo una vez a la semana (2). Esto muy probablemente se debe a que no son del agrado de los niños, como ellos mismos refieren, a costumbres adquiridas en la familia, o a los escasos recursos económicos que impiden su adquisición.

Es de destacar que más de la mitad de los niños que tenían valores superiores al percentil 90 de Hcy indicaron un consumo de carne de 1 a 2 veces por semana. Por lo tanto, la HHcy podría estar relacionada con una mayor síntesis de Hcy a partir de la ingesta de la metionina presente en la carne (29). Sin embargo, se necesitan estudios con mayor población que evalúen si en población infantil colombiana la ingesta alta de proteínas de origen animal está relacionada con el aumento de Hcy, como ya se ha demostrado en adultos paquistaníes (30).

En el presente estudio se observó baja prevalencia de sobrepeso y obesidad, datos que difieren de los reportados por el ENSIN (2010) en el que se obtuvieron mayores frecuencias para estas dos variables. Esta diferencia podría originarse en estilos de vida diferentes y permite sugerir realizar estudios complementarios que aborden estos aspectos.

Con respecto a la relación entre homocisteína y obesidad, y a diferencia de lo observado en Brasil (31), en el presente estudio no se encontró asociación significativa entre obesidad y altas concentraciones de Hcy ni entre hiperhomocisteine- mia y otras variables antropométricas. Así mismo, son opuestos a lo encontrado en niños obesos de Australia en quienes se demostró correlación entre hiperhomocisteínemia, IMC, masa grasa y porcentaje de grasa (32). Sin embargo, como se mencionó antes, es necesario realizar estudios que incluyan más población.

El promedio de triglicéridos en la población analizada fue mayor en nińas que en nińos, con significancia mayor entre los 6 y los 9 ańos. Estos datos coinciden con lo reportado en dos estudios realizados en población infantil colombiana (33, 34). De la misma manera, el c-VLDL presentó concentraciones más altas en niñas que en niños. Esta relación es similar a la encontrada por Bercedo y colaboradores, en niños españoles con edades entre 8 y 15 años de edad, donde las niñas tuvieron valores más altos de c-VLDL que las niños (35). El valor promedio de colesterol total para la población estudiada $(170 \mathrm{mg} / \mathrm{dL})$ fue mayor al encontrado en la ciudad de Belém, Brasil $(142 \mathrm{mg} /$ dL) (31). Esta diferencia puede deberse a que la escuela brasileńa era de carácter privado en comparación con el colegio Manuel Elkin Patarroyo y el colegio Manuel Elkin Patarroyo. Así mismo, no se encontró correlación entre homocisteína, perfil lipídico e hipertensión arterial para el momento de la toma de muestra. Sin embargo, datos encontrados en Polonia ponen de manifiesto una relación entre hiperhomocisteinemia, perfil lipídico e hipertension arterial en nińos (36).

En la presente investigación no hubo asociación entre los niveles de homocisteína y otros factores de riesgo cardiovascular, de forma homóloga a estudios realizados por Aboul Ella en adolescentes egipcios donde se expone que el nivel de homocisteína no tiene una influencia marcada con otros factores de riesgo cardiovascular como el perfil lipídico, la glicemia y el IMC (37).

Sin embargo, otros estudios reportan de manera contradictoria una asociación positiva de la Hcy con otros factores de riesgo cardiovascular como 
los hábitos alimenticios, el IMC, el porcentaje de grasa corporal, historial familiar de enfermedad cardiaca, entre otros (38-43). Estos resultados permiten intuir que aún existe controversia sobre el tema, lo que hace necesario seguir investigando sobre los niveles de homocisteína en niños que posean otros factores de riesgo cardiovascular.

Los resultados del presente trabajo permiten concluir que aunque en la población estudiada la hiperhomocisteinemia no está asociada con otros factores de riesgo cardiovascular, la presencia de alteraciones en el perfil lipídico, antropometría y estilos de vida de algunos de los niños hace necesario implementar programas de promoción y prevención de la salud que permitan la modificación de hábitos inadecuados y la implementación de conductas saludables de vida que contribuyan a disminuir el riesgo cardiovascular al que está expuesto la población.

\section{Conflictos de interés}

Las autoras declaran no tener ningún conflicto de interés.

\section{Agradecimientos}

Las autoras expresan su agradecimiento a:

Las Dras. Judith Huérfano, Olga Lucía Orjuela y María Vilma Giratá Pedraza por su acompañamiento y colaboración en el proceso de recolección de las muestras.

Las directivas del colegio IED Manuel Elkin Patarroyo, por permitir la participación de sus estudiantes para la realización de este estudio y facilitar el acceso de los investigadores a sus instalaciones.

Los estudiantes y padres de familia del colegio IED Manuel Elkin Patarroyo que participaron en el presente estudio; por su asistencia y disponibilidad.
La docente Clara Pardo, coordinadora del colegio IED Manuel Elkin Patarroyo, por su comprensión y apoyo.

Los Laboratorios Annar Diagnóstica Import y Becton Dickinson de Colombia, por el apoyo brindado en equipos y reactivos.

\section{Referencias}

1. Guías Colombianas de Cardiología Síndrome Coronario Agudo sin elevación del ST (angina inestable e infarto agudo del miocardio sin elevación del ST. Disponible en http://scc.org. co/wp-content/uploads/2012/08/8-guia-enf-coronaria-2008. pdf

2. Instituto Colombiano de Bienestar Familiar. Encuesta Nacional de la Situación Nutricional en Colombia. Resumen Ejecutivo ENSIN ed. Bogotá: ICBF; 2010. p. 92-3.

3. Graham IM, Daly LE, Refsum HM, Robinson K, Brattstrom LE, Ueland PM, et al. Plasma homocysteine as a risk factor for vascular disease. The European Concerted Action Project. JAMA. 1997;277(22):1775-81.

4. Marie SK, Shinjo SK, Oba-Shinjo SM, da Silva R, Barbosa $\mathrm{KC}$, Yamamoto F, et al. Methylenetetrahydrofolate reductase gene polymorphism is not related to the risk of ischemic cerebrovascular disease in a Brazilian population. Clinics (Sao Paulo). 2007;62(3):295-300.

5. Deregibus M, Haag D, Ferrario C, Grunfeld B, Miceli I, Briones $\mathrm{L}$, et al. Consenso sobre factores de riesgo de enfermedad cardiovascular en pediatría: Hipertensión arterial en el niño y el adolescente. Arch Argent Pediatr. 2005;103:348-57.

6. Vera-Delgado A. Factores de riesgo cardiovascular: guías de prevención primaria (una propuesta). Revista Colombiana de Cardiología. 2008;15:149-52.

7. Hernández MA, García HL. Factores de riesgo y protectores de enfermedades cardiovasculares en población estudiantil universitaria. Rev. Fac. Med. 2007;30:119-23.

8. Munuera JN. Psicología para Ciencias de la Salud: Estudio Del Comportamiento Humano Ante la Enfermedad: McGraw-Hill / Interamericana de España, S.A.; 2004.

9. Babor TF, Higgins-Biddle JC, Saunders JB, Monteiro MG. Cuestionario de Identificación de los Transtornos debidos al Consumo de Alcohol. Organizacion Mundial de Salud. Ginebra: Suiza; 2001. p. 30-2.

10. Heatherton T KL, Frecker R, F. The Fagerström test for nicotine dependence: a review of the Fagerström tolerance questionnaire. Br J Addiction 1991;86:1119-27.

11. García P, Urrego J, D’Achiardi R, Delgado V. Hipertensión arterial: diagnóstico y manejo. Univ Sci. 2005;45(2):77-84. 
12. Mancia G, Backer GD, Dominiczak A, Cifkova R, Fagard $\mathrm{R}$, Germano $\mathrm{G}$, et al. Guías de práctica clínica para el tratamiento de la hipertension arterial 2007. Rev Esp Cardiol. 2007;60(9):968.

13. Organización Mundial de Salud. Enfermedades Cardiovasculares. Datos y Cifras. Ginebra, 2012 [citado el 20 de Febrero de 2012]; Disponible en: http://www.who.int/mediacentre/ factsheets/fs317/es/index.html.

14. Instituto nacional de corazón, pulmón y sangre de Estados Unidos. Programa Nacional e Internacional de Educación sobre colesterol para Niños de Estados Unidos USA. 2005 [citado el 12 de Febrero 2012]; Disponible en: https://www.nhlbi. nih.gov/about/ncep/ncep_pd.htm.

15. Asociación-Española-de-Pediatría-de-Atención-Primaria. Guía Breve para la promoción de la salud cardiovascular en la infancia y adolescencia 2005 [citado el 20 de Febrero de 2012]. Disponible en: http://www.aepap.org/pdf/guia_cardiovascular.pdf.

16. Ariza IDS, Bustos EGd. Guía de diagnóstico y control de la diabetes mellitus. Asociación Latinoamericana de Diabetes (ALAD). 2013.

17. Organización Mundial de la Salud. Riesgos para la salud de los jóvenes. 2011 [citado el 22 de Septiembrede 2014]; Disponible en: http://www.who.int/mediacentre/factsheets/fs345/es/.

18. Shield K, Monteiro M, Roerecke M. Alcohol consumption and burden of disease in the Americas in 2012: implications for alcohol policy. Revista Panamericana Salud Publica 38(6)2015. Disponible: http://iris.paho.org/xmlui/bitstream/ handle/123456789/18558/v38n6a2_442-449.pdf?sequence $=1 \&$ isAllowed $=y$

19. Pérez M, Pinzón H. Uso del tabaco entre los jóvenes colombianos. Retos para los profesionales en salud pública. Salud Uninorte. 2005;21(0120-4442):66-75.

20. Fernández C. El trago destruye las facultades cognitivas de nińos y adolescentes. El tiempo; 2015 [citado el 1 de Febrero de 2016]; Disponible en: http://www.eltiempo.com/estilo-de-vi$\mathrm{da} /$ salud/consumo-de-licor-en-ninos/16456346.

21. UNESCO. Educación para la salud. Promoción y educación para la salud. Hamburgo, 1997 [citado el 22 de Febrero de 2012]. disponible en: http://www.unesco.org/education/uie/ confintea/pdf/6b_span.pdf

22. Miguel Cabanillas EL. Educación para la salud. Guía práctica para promover estilos de vida saludables. Piramides E, editor 2008.

23. Villarreal E, Forero Y, Poveda E, Baracaldo C, López E. Marcadores de riesgo cardiovascular en escolares de cinco departamentos de la región oriental en Colombia. Biomédica. 2008;28:38-49.

24. Davila-Rodriguez MI, Torres-De la Cruz VM, Novelo-Huerta HI, Said-Fernandez S, Cerda-Flores RM, Cortes-Gutierrez EI. Total homocysteine levels in healthy children from the Monterrey metropolitan area, Mexico. Prague Med Rep. 2010;111(2):135-41.
25. Maximova K, Kuhle S, Davidson Z, Fung C, Veugelers PJ. Cardiovascular Risk Factor Profiles of Normal and Overweight Children and Adolescents: Insights From the Canadian Health Measures Survey. Canadian Journal of Cardiology. (0).

26. Ganji V, Kafai M. Population references for plasma total homocysteine concentrations for U.S. children and adolescents in the post-folic acid fortification era. J Nutr. 2005;135(9):22536. Epub AT- 2005/09/06 09:00 MHDA- 2005/12/13 09:00.

27. Van Beynum IM, den Heijer M, Thomas CMG, Afman L, Oppenraay-van Emmerzaal D, Blom HJ. Total homocysteine and its predictors in Dutch children. The American Journal of Clinical Nutrition. 2005;81(5):1110-6.

28. Nemec V, Botkayova E, Kutilek S. Serum homocysteine levels in Czech children and adolescents. Acta Medica (Hradec Kralove). 2012;55(2):87-90.

29. Blom HJ, Smulders Y. Overview of homocysteine and folate metabolism. With special references to cardiovascular disease and neural tube defects. J Inherit Metab Dis. 2011;34(1):7581.

30. Yakub M, Iqbal Mp Fau - Iqbal R, Iqbal R. Dietary patterns are associated with hyperhomocysteinemia in an urban Pakistani population. J Nutr. 2010;140(7):1261-6.

31. Ribas SA, Silva LCSd. Dislipidemia em escolares na rede privada de BelÃ@m. Arquivos Brasileiros de Cardiologia. 2009;92:446-51.

32. Xanthakis V, Enserro DM, Murabito JM, Polak JF, Wollert $\mathrm{KC}$, Januzzi JL, et al. Ideal cardiovascular health: associations with biomarkers and subclinical disease and impact on incidence of cardiovascular disease in the Framingham Offspring Study. Circulation. 2014;130(19):1676-83. Epub 2014/10/03

33. Poveda E, Callas N, Baracaldo C, Castillo C, Hernández P, Guerra M. Evaluación de las concentraciones de lípidos y apoproteínas A-I y B-100 en un grupo de escolares de cinco departamentos del centro-oriente de Colombia. Biomédica. 2007;27:385-99.

34. Uscátegui R, Uribe MÁ, Salinas IL, Terranova WS, Maluendas LM, Arteaga RA, et al. Factores de riesgo cardiovascular en niños de 6 a 18 años de Medellín (Colombia). An Pediatr 2003;58 (05):411-7.

35. Bercedo-Sanz A, González-Lamuño D, Cacho PM, Molera MA, Rey JCR, Fernández SB, et al. Asociación entre el perfil lipídico y genotipo de la apoproteína E en niños españoles (815 años). Asoc Esp Ped. 1998;49(2):120-4.

36. Glowinska B, Urban M, Koput A, Galar M. New atherosclerosis risk factors in obese, hypertensive and diabetic children and adolescents. Atherosclerosis. 2003;167(2):275-86. Epub 2003/06/24

37. Aboul Ella NA, Shehab DI, Ismail MA, Maksoud AA. Prevalence of metabolic syndrome and insulin resistance among Egyptian adolescents 10 to 18 years of age. J Clin Lipidol. 2010;4(3):185-95. 
38. Casanueva E V, Cid C X, Cancino M M, Borzone T L, Cid S L. Homocisteína en niños y adolescentes. Relación con historia familiar de enfermedad cardiovascular. Revista médica de Chile. 2003;131:997-1002.

39. Greenlund KJ, Srinivasan SR, Xu JH, Dalferes E, Jr., Myers L, Pickoff A, et al. Plasma homocysteine distribution and its association with parental history of coronary artery disease in black and white children: the Bogalusa Heart Study. Circulation. 1999;99(16):2144-9.

40. Kosch A, Koch HG, Heinecke A, Kurnik K, Heller C, Nowak-Gottl U. Increased fasting total homocysteine plasma levels as a risk factor for thromboembolism in children. Thromb Haemost. 2004;91(2):308-14.

41. Papandreou D, Malindretos P, Arvanitidou M, Makedou A, Rousso I. Homocysteine lowering with folic acid supplements in children: effects on blood pressure. Int J Food Sci Nutr. 2010;61(1):11-7.

42. Almonacid CA, Camarillo MS, Gil Z, Medina CY, Rebellón JN, Mendieta H. Evaluación de factores de riesgo asociados a enfermedad cardiovascular en jovenes universitariosde la localidad Santa Fé en Bogotá, Colombia. NOVA. 2016; 14 (26); $35-45$.

43. Flórez, R. A. N. Avances y perspectivas en Síndrome de Asperger. 2014; Nova, 12(21). 